13.2

\title{
Анализ поведения индивидуальных эмиссионных центров на поверхности многоострийного полевого катода
}

\author{
() А.Г. Колосько, Е.О. Попов, С.В. Филиппов \\ Физико-технический институт им. А.Ф. Иофрфе РАН, \\ Санкт-Петербург, Россия \\ E-mail: agkolosko@mail.ru
}

Поступило в Редакцию 13 декабря 2018 г.

В окончательной редакции 13 декабря 2018 г.

Принято к публикации 20 декабря 2018 г.

\begin{abstract}
Представлена методика анализа поверхности многоострийных полевых катодов на основе данных компьютеризированного полевого эмиссионного проектора и системы быстрого сканирования высоким напряжением. Результаты исследований нанокомпозитного эмиттера полимер/углеродные нанотрубки позволили получить распределение эмиссионных центров по поверхности катода, а также оценить изменение работы выхода отдельных эмиссионных центров при разных уровнях приложенного напряжения. Проведена численная оценка вклада отдельных групп эмиссионных центров в изменение формы вольт-амперной характеристики.
\end{abstract}

DOI: 10.21883/PJTF.2019.06.47504.17643

Полевые эмиттеры являются ярким примером применения квантовых эффектов в современной электронике [1]. Использование в качестве полевого эмиттера материалов с нано- и микроструктурированной поверхностью позволяет существенно повысить предел достижимых токов, а также стабильность работы эмиттера [2]. Однако стохастическое взаимодействие нанои микроострий на его поверхности с заряженными и нейтральными частицами остаточной атмосферы приводит к труднопрогнозируемому поведению системы в условиях технического вакуума $\left(\sim 10^{-7}\right.$ Torr $)$. Поэтому множество научных работ направлено на изучение адсорбционно-десорбционных процессов на поверхности полевых эмиттеров и влияния этих процессов на макроскопические характеристики, такие как вольт-амперная характеристика (BAX) и временна́я стабильность эмиссионного тока. Самым эффективным инструментом для наблюдения изменения свойств поверхности эмиттера в ходе его работы является полевой эмиссионный проектор с люминесцентным экраном и цифровой видеокамерой, который регистрирует распределение тока на поверхности эмиттера в виде пятнистых картин свечения [3].

Можно выделить общие закономерности взаимодействия многоострийных эмиттеров с частицами остаточной атмосферы. В работе [4] наблюдалось стохастическое изменение картины свечения эмиттера, состоящего из графеновых волокон на кремниевой подложке. Серия картин, полученная в ходе длительной работы эмиттера ( $~ 3 \mathrm{~h})$ при постоянном напряжении, показала, что в целом распределение центров на поверхности не меняется, но наблюдается изменение их числа, а некоторые центры меняют свое положение и уровень тока. Эти флуктуации объяснялись бомбардировкой центров ионами остаточной атмосферы. Изменение же уровня напряжения приводило к увеличению числа эмиссионных центров и увеличению яркости общей картины. В работе [5] было исследовано влияние уровня вакуума на полевую эмиссию графеновых нанопленок. Ухудшение вакуумных условий на картинах свечения приводило к уменьшению концентрации эмиссионных центров, а при улучшении они частично восстанавливались. Это изменение объяснялось адсорбцией на центры эмиссии атомов кислорода, которые увеличивают работу выхода электронов. В [6] была изучена поверхность нанокомпозита с углеродными нанотрубками (УНТ). На увеличенном изображении отдельных УНТ наблюдались светящиеся пятна, которые авторы связали с адсорбированными частицами, которые локально улучшают полевую эмиссию. При нагреве до $900 \mathrm{~K}$ мерцание и перемещение светящихся пятен на общей картине свечения прекращалось. При охлаждении же катода адсорбаты вновь заполняли поверхность нанокомпозита. Время возврата эмиттера в исходное состояние при резком охлаждении до $300 \mathrm{~K}$ составляло $\sim 1 \min$ при давлении $10^{-8}$ Torr.

Цель настоящей работы состоит в получении эмиссионного профиля полевого эмиттера новым методом компьютерной обработки картин свечения, а также в изучении связи макроскопических характеристик эмиттера с локальными изменениями работы выхода отдельных эмиссионных центров на его поверхности.

Экспериментальный стенд состоит из компьютеризированной установки, которая позволяет регистрировать и обрабатывать в режиме онлайн вольт-амперные характеристики полевых эмиттеров [7]. ВАХ получаются в режиме быстрого сканирования: полусинусоидальный импульс амплитудой $U_{m} \sim 1 \mathrm{kV}$ дает одну ВАХ каждые $20 \mathrm{~ms}$. Также стенд включает в себя полевой эмиссионный проектор с онлайн обработкой картин свечения [8]. Уровень вакуума в измерительной камере 


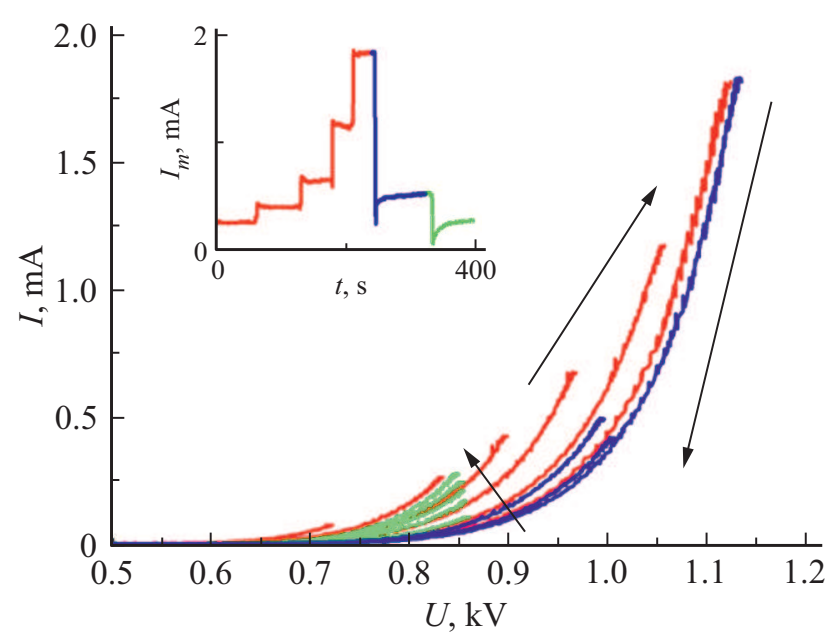

Рис. 1. Изменение формы BAX полевого катода при росте, падении и постоянном уровне амплитуды импульсов напряжения $U_{m}$. На вставке показано изменение амплитуды импульсов эмиссионного тока $I_{m}$ со временем.

поддерживается на уровне $\sim 10^{-7}$ Torr. Межэлектродное расстояние (расстояние между поверхностью металлической подложки эмиттера и люминофорным экраном) устанавливалось равным $170 \mu \mathrm{m}$.

Исследованный образец представлял собой нанокомпозит полимер/одностенные УНТ, который был изготовлен методом вращающегося столика из суспензии полимер-УНТ-о-ксилол. Данный вид эмиттеров имеет определенные преимущества перед эмиттерами из нанотрубок без полимерной матрицы [9].

Методика анализа картин свечения с получением гистограммы максимальных токов локальных эмиссионных центров, а также гистограммы соответствующих коэффициентов усиления поля была подробно описана в работах $[10,11]$. Она реализуется в несколько этапов: 1) сбор координат и размеров зон флуктуации эмиссионных центров методом поиска точек максимальной яркости на картинах свечения - порядка $100 \mathrm{~s}$ до выхода на уровень насыщения числа найденных центров; 2) слежение за яркостью найденных зон - порядка $100 \mathrm{~s}$ до выхода на уровень насыщения суммарной максимальной яркости; 3) получение значений локальных токов путем распределения измеряемого тока (амплитуды импульсов $I_{m}$ ) по эмиссионным центрам в пропорции к их яркости; 4) расчет коэффициентов усиления поля центров $\beta$ по их максимальным локальным токам методом численного решения уравнения Фаулера-Нордгейма при заданных работе выхода $\varphi$ (была выбрана равной $4.6 \mathrm{eV}$, что соответствует состоянию нанотрубки без адсорбатов $[12]$ ) и площади эмиссии каждого центра $S_{0}$ (была выбрана равной $5 \mathrm{~nm}^{2}$, что соответствует компьютерным расчетам [13]). Для анализа и сравнения получаемых распределений нами была написана отдельная программа.
На рис. 1 представлено поведение ВАХ при ступенчатом росте и падении амплитуды импульсов напряжения $U_{m}$. С ростом $U_{m}$ BAX смещается в сторону меньших токов, что указывает на изменение адсорбционнодесорбционного баланса в сторону ухудшения эмиссионной способности центров, которое может быть связано с электронно-стимулированной десорбцией частиц $\mathrm{CO}_{2}$ с поверхности анода [14]. Уменьшение $U_{m}$ приводит к уменьшению ВАX без изменения положения, т.е. десорбция частиц с поверхности эмиттера происходит не сразу. Последующая выдержка при низких $U_{m}$ приводит к плавному росту эмиссионного тока со временем. Это может быть объяснено изменением работы выхода эмиссионных центров в результате десорбции с них частиц $\mathrm{CO}_{2}$. При циклическом изменении напряжения форма BAX меняется также циклически, что исключает существенное влияние деструктивных процессов на поверхности эмиттера.

На рис. 2 представлена зависимость от времени уровня тока $I_{m}$ при ступенчатом уменьшении, а затем росте $U_{m}$. Суммарная яркость центров эмиссии, зарегистрированных полевым проектором, линейно зависит от уровня тока, что позволяет использовать ее для расчета локальных эмиссионных токов и последующего вычисления коэффициентов усиления поля $\beta$. Полученные для каждой ступени напряжения гистограммы представлены на рис. $3, a$. Диапазоны значений $\beta$ у рассчитанных гистограмм с хорошей точностью совпали почти для всех ступеней напряжения. Однако гистограмма самой нижней ступени показала завышенные значения. Поскольку физически коэффициент усиления поля обусловлен формой поверхности катода, завышенные значения $\beta$, скорее всего, связаны с уменьшением работы выхода части эмиссионных центров. Анализ отдельных центров показал, что уменьшение это составляет $\sim 0.3 \mathrm{eV}$, что соответствует литературным данным, описывающим влияние адсорбатов $\mathrm{CO}_{2}$ [15]. Исходя из последнего

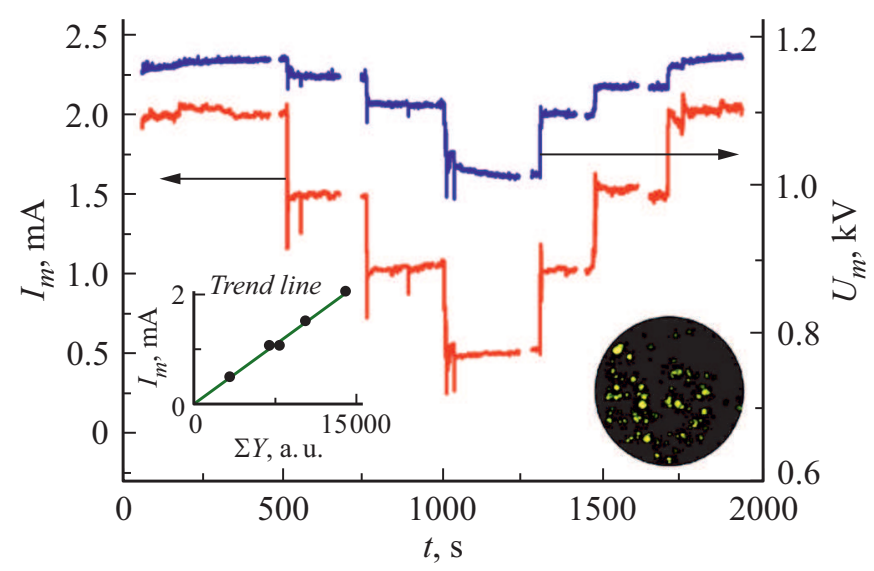

Рис. 2. Зависимость амплитуды импульсов эмиссионного тока $I_{m}$ и амплитуды импульсов приложенного напряжения $U_{m}$ от времени. На вставках показаны картина свечения, зарегистрированная при $I_{m}=2 \mathrm{~mA}$ (справа) и зависимость уровня тока от суммарной яркости центров (слева). 

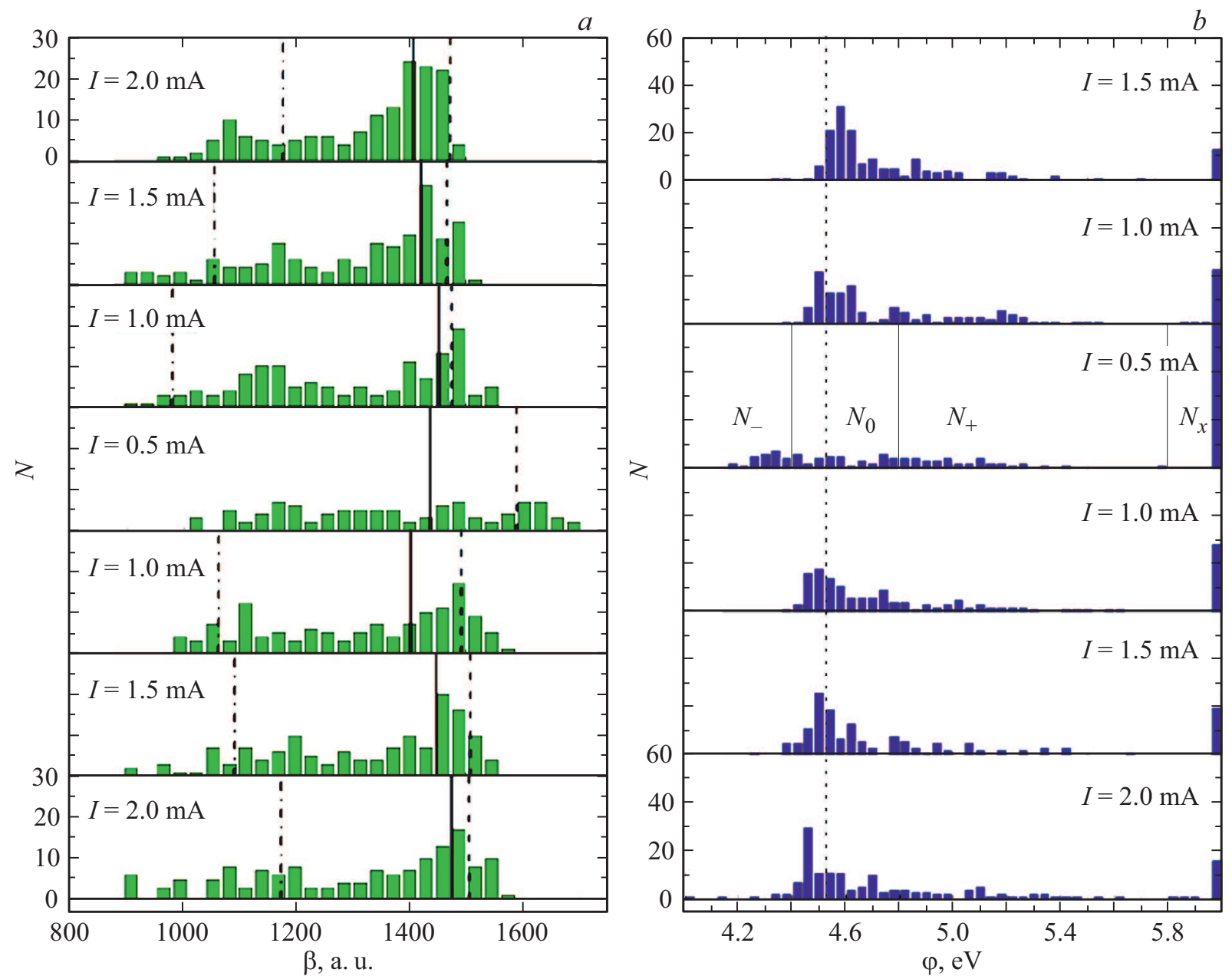

Рис. 3. Гистограммы характеристик эмиссионных центров при разных уровнях тока: $a-$ коэффициента усиления поля $\beta$; $b-$ работы выхода $\varphi$. Вертикальными линиями отмечены значения $\beta$ для различных представителей групп: самых ярких центров эмиссии (штриховая), самых стабильных (сплошная) и интенсивно гаснущих (штрихпунктирная). Пунктиром показано исходное значение работы выхода $(4.6 \mathrm{eV}$ для центров первой ступени). Всего центров в анализе 160 .

предположения мы фиксировали коэффициенты $\beta$, полученные на ступени с максимальным $U_{m}$, и анализировали соответствующее изменение $\varphi$ эмиссионных центров при других уровнях напряжения (рис. $3, b)$.

У части центров при изменении $U_{m}$ работа выхода оставалась постоянной $(\varphi=4.6 \pm 0.2 \mathrm{eV})$. Доля таких центров на ступени с минимальным $U_{m}$ составила $N_{0} / N=24 \%$, а их относительный вклад в общий ток ступени с максимальным $U_{m}-I_{0} / I=31 \%$. Работа выхода $\varphi$ других центров уменьшилась $(<4.4 \mathrm{eV})$, их доля $N_{-} / N=16 \%$, а вклад в общий ток $I_{-} / I=28 \%$. Для третьей группы значение $\varphi$ увеличилось $(>4.8 \mathrm{eV})$, в этом случае $N_{+} / N=23 \%$ и $I_{+} / I=24 \%$. Остальные центры вышли из процесса эмиссии, уменьшив свою яркость ниже порога регистрации: $N_{X} / N=28 \%$ и $I_{X} / I=17 \%$. Скачкообразное уменьшение работы выхода некоторых центров от ступени к ступени, возможно, связано с недостаточно высоким разрешением USBмикроскопа ( 15 $\mu$ m на пиксель) и наложением области свечения нескольких центров друг на друга, так что в одном зарегистрированном центре могло оказаться несколько отдельных микроскопических источников эмиссии. Их поочередное покрытие адсорбатами могло привести к переключению внимания программы с одного пика яркости на другой.

Отметим, что уменышение работы выхода происходит в основном у центров с наибольшими $\beta$, тогда как центры с наименьшими $\beta$, наоборот, стремятся увеличить свою работу выхода $\varphi$. Такая сепарация может быть связана с тем, что центры с наибольшими $\beta$ испытывают повышенную токовую нагрузку и достаточно сильно разогреты для освобождения от адсорбатов. В то время как „слабые“ эмиссионные центры, наоборот, подвержены осаждению на них дополнительных частиц при пониженных токах.

Основной вклад в увеличение тока эмиссии $(+430 \mu \mathrm{A})$ относительно уровня, ожидаемого при понижении напряжения, вносят самые яркие центры, однако им в противовес уменьшает ток $(-380 \mu \mathrm{A})$ значительное количество тусклых центров. Таким образом, за фор- 
мирование новой ВАХ отвечает весь ансамбль эмиссионных центров, перестраиваясь под воздействием нового адсорбционно-десорбционного баланса. Отметим, что величина стартовой $\varphi(4.6 \mathrm{eV})$ почти не влияет на вид гистограмм, т.е. изменение работы выхода центров на $\sim 0.3 \mathrm{eV}$ не зависит от выбранной модели: десорбция $\mathrm{CO}_{2}$ при понижении напряжения или, например, адсорбция $\mathrm{H}_{2}$ либо $\mathrm{H}_{2} \mathrm{O}$.

Проведенный анализ показал возможность оценки изменения работы выхода отдельных центров эмиссии и определения их вклада в изменение макроскопического уровня эмиссионного тока, который определяет форму ВАХ. Возможность одновременного изучения свойств множества отдельных эмиссионных центров и построение их общей статистики открывает новые возможности для проверки теории полевой эмиссии, а также для моделирования макроскопических свойств катода на основе кратковременных измерений.

\section{Список литературы}

[1] Егоров Н.В., Шешин Е.П. Автоэлектронная эмиссия. Принципы и приборы. Долгопрудный: Интеллект, 2011. $703 \mathrm{c}$.

[2] Соминский Г.Г., Тумарева Т.А. // Изв. вузов. Прикладная нелинейная динамика. 2015. Т. 23. № 2. С. 74-93.

[3] Baturin S.S., Baryshev S.V. // Rev. Sci. Instr. 2017. V. 88. N 3. P. 033701.

[4] Khare R., Shinde D.B., Bansode S., More M.A., Majumder M., Pillai V.K., Late D.J. // Appl. Phys. Lett. 2015. V. 106. N 2. P. 023111.

[5] Васильева Е.А., Клещ, В.И., Образиов А.Н. // ЖТФ. 2012. T. 82. B. 7. C. $107-111$.

[6] Dean K.A., Chalamala B.R. // J. Appl. Phys. 1999. V. 85. N 7. P. 3832-3836.

[7] Попов Е.О., Колосько А.Г., Филиппов С.В., Романов П.А., Федичкин И.Л. // Наноматериалы и наноструктуры - XXI век. 2016. № 1. С. 14-26.

[8] Popov E.O., Kolosko A.G., Filippov S.V., Romanov P.A., Fedichkin I.L. // Mater. Today. Proc. 2018. V. 5. N 5. Pt 3. P. 13800-13806.

[9] Pandey A., Prasad A., Moscatello J.P., Engelhard M., Wang C., Yap Y.K. // ACS Nano. 2013. V. 7. N 1. P. 117-125.

[10] Filippov S.V., Popov E.O., Kolosko A.G., Vinnichek R.N. // J. Phys.: Conf. Ser. 2017. V. 917. N 9. P. 092022.

[11] Popov E.O., Kolosko A.G., Filippov S.V., Terukov E.I. // J. Vac. Sci. Technol. B. 2018. V. 36. N 2. P. $02 C 106$.

[12] Gao R., Pan Z., Wang Z. // Appl. Phys. Lett. 2001. V. 78. N 12. P. 1757-1759.

[13] Popov E.O., Filippov S.V., Kolosko A.G., Romanov P.A. // Int. Vac. Nanoelectron. Conf. Techn. Digest. Kyoto, Japan: 2018. P. 256.

[14] Popov E.O., Kolosko A.G., Filippov S.V., Romanov P.A., Terukov E.I., Shchegolkov A.V., Tkachev A.G. // Appl. Surf. Sci. 2017. V. 424. Pt 2. P. 239-244.

[15] Strasser G., Bertel E., Netzer F.P. // J. Catal. 1983. V. 79. N 2. P. $420-430$. 